\title{
EL MERCADO LABORAL EN EL EJERCICIO DE LA PROFESIÓN: LA EXPERIENCIA DE ESTUDIANTES DE MAESTRÍA DE NEGOCIOS DE UNA UNIVERSIDAD COSTARRICENSE
}

\author{
SAÚL NÚÑEZ CORTÉS \\ Escuela Ciencias de la Administración, Universidad Estatal a Distancia, Costa Rica \\ Escuela de Ciencias Económicas y Empresariales, Universidad Latinoamericana \\ de Ciencia y Tecnología (ULACIT), Costa Rica \\ saulnunez@costarricense.cr
}

\section{RESUMEN}

El objetivo de este estudio es identificar los principales aspectos relacionados con el mercado laboral de estudiantes de maestría de la carrera de Negocios de la Universidad Latinoamericana de Ciencia y Tecnología (ULACIT), quienes simultáneamente han trabajado y estudiado durante el 2012. La investigación hace referencia a perspectivas que relacionan el "mundo del trabajo" y las universidades. El estudio es de corte descriptivo, los datos son analizados y discutidos tal y como suceden. Se empleó una muestra de 50 estudiantes de posgrado de Negocios de ULACIT, a la cual se aplicó un cuestionario para recoger la información. Los resultados muestran que los estudiantes poseen oportunidad de encontrar empleo y desarrollarse profesionalmente en cargos gerenciales dentro del mercado laboral costarricense. La experiencia, el liderazgo y el trabajo en equipo son factores importantes para lograr el éxito laboral. La bolsa de empleo sigue siendo el medio de búsqueda de empleo tradicional para dichos estudiantes mientras que la entrevista y la revisión del currículo son el método de selección de personal más utilizado por las empresas. Las diferencias salariales se relacionan con los niveles de experiencia laboral y no con el grado académico universitario.

PALABRAS CLAVE: MERCADO LABORAL, OPORTUNIDAD DE EMPLEO, CARGOS Y ACTIVIDADES, MEDIO DE BÚSQUEDA DE EMPLEO, MÉTODO DE SELECCIÓN DE PERSONAL, INGRESO Y FORMACIÓN.

\section{ABSTRACT}

The objective of this work is to identify the main aspects related with the labor market of MBA students of the business career ULACIT who have worked and studied at the same time during the 2012. Research refers to perspectives that relate the "world of work" and the universities, whose contributions have been used to carry out studies to postgraduate level. The study is carried out within a type of descriptive research whose data are analyzed and discussed as they occur. Within the research has been used a sample of 50 graduate students from ULACIT business, which has been implemented a questionnaire for the coIlection of data related to the objectives of research raised here. The results show that students at the masters of business of ULACIT have opportunity to find employment and to develop professionally in managerial positions within the Costa Rican labor market. The experience, leadership and teamwork are important factors to succeed in the labor market. The bag of employment remains the medium of traditional job search for these students while the interview and review of the curriculum are the method of selecting staff more used by the companies. The differences between what you earn a person of another is oriented in the levels of work experience with the students and not by his college career at the graduate level.

KEYWORDS: LABOR MARKET, JOB OPPORTUNITY, POSITIONS AND ACTIVITIES, THROUGH JOB SEARCH, RECRUITMENT METHOD, INCOME AND EDUCATIONAL TRAINING. 


\section{INTRODUCCIÓN}

Con frecuencia, se escuchan lamentos dentro de las aulas universitarias y las salas ejecutivas, sobre lo difícil del mercado laboral actual. En efecto, es una realidad que afecta a los estudiantes de maestría de Negocios, como a muchos otros, que temen sobre el futuro de su desarrollo profesional.

Según la Encuesta Continua de Empleo (ECE) del Instituto Nacional de Estadística y Censos (INEC) del segundo trimestre de 2012, la cantidad de personas desempleadas fue de $222 \mathrm{mil}$ personas, con un incremento absoluto casi de 30 mil personas respecto al segundo trimestre del 2011. Sin embargo, la tasa de desempleo de 10,2\% en el segundo trimestre 2012 es únicamente 0,4 puntos porcentuales mayores a la del mismo trimestre del 2011 (9,8\%), debido al fuerte incremento del empleo incrementa de manera importante la fuerza de trabajo.

Por otro lado, según la encuesta de Manpower Group sobre escasez de talento (2012), el hecho de que las empresas no obtienen el recurso humano requerido es una situación preocupante ante un país que tiene una tasa de desempleo del 10,2\% para el segundo trimestre del 2012.

Al respecto, Manpower Group encuestó a 10 232 empleadores en la región de América, cubriendo Argentina, Brasil, Canadá, Colombia, Costa Rica, EE.UU., Guatemala, México, Panamá y Perú durante el primer trimestre de 2012. Y como resultado, el $41 \%$ mencionó la dificultad de encontrar los candidatos adecuados para cubrir sus puestos vacantes, por encima del 37\% en 2011 y 34\% en 2010.

Con cifras alarmantes de este tipo, una vez que las personas son aceptadas para ingresar a la universidad, ya sea que trabajen o estudien, o lo hagan simultáneamente, es de vital importancia que conozcan lo que acontece sobre el merca- do laboral donde desean desarrollarse profesionalmente.

Merece entonces plantearse la siguiente pregunta de investigación:

¿Cuáles son los principales aspectos relacionados con el mercado laboral para los estudiantes de maestría de la carrera de Negocios de la ULACIT quienes simultáneamente han trabajado y estudiado durante el 2012?

Por tanto, el principal objetivo de esta investigación es precisamente identificar los aspectos relevantes relacionados con el mercado laboral de estudiantes de maestría de la carrera de Negocios quienes han trabajado y estudiado simultáneamente durante el 2012, con respecto a lugares generadores de empleo, actividades que se realizan, formas para buscar empleo, métodos de selección de empleados, ingresos, y áreas claves de formación requeridas en el desarrollo profesional.

Para lograr el objetivo, se realizó una investigación con un enfoque mixto (cualitativo y cuantitativo), el cual presenta un diseño no experimental transaccional con alcance de tipo descriptivo. Se aplicó un cuestionario a 50 estudiantes de maestría.

La muestra está conformada por alumnos que se desarrollan profesionalmente en instituciones públicas y empresas privadas de carácter nacional y firmas transnacionales de gran trayectoria con operaciones en Costa Rica.

la investigación no pretende generar información específica de ningún área o énfasis de los estudiantes de maestría de Negocios de ULACIT, sino elaborar un documento relevante con información de contexto general sobre el mercado laboral de la muestra en estudio. Es decir, el estudio pretende generar datos que constituyan la materia prima para investigaciones más precisas y despierte el interés en nuevas líneas de 
investigación sobre estudios de seguimiento de egresados.

Los resultados de esta investigación serán de interés para futuros estudiantes de maestría en negocios que están o desean ingresar al mercado laboral, y que muestran especial atención a la interrelación de los elementos que componen el mercado, los cuales generan información útil para enfrentar con inteligencia los retos de una de las áreas de mayor importancia en el desarrollo del país.

\section{Aspectos teóricos}

Según Lobo y Morua (2011), los aportes de Harald Schomburg en relación con el "mundo del trabajo" aseveran que la educación superior muestra las siguientes funciones:

- Función de preparación o entrenamiento

- Función de socialización

- Función de distribución de estatus o posición social (p. 53)

Con respecto a las afirmaciones de Teichler en lo relativo al mundo del trabajo, la educación posee dos funciones primordiales:

- La primera es una función preparadora que tiene que ver con las capacidades cognitivas y posiblemente efectivas y senso-motrices que puede ser útiles para hacer frente a las tareas del trabajo.

- En segunda instancia, se halla la función distribuidora de status donde el nivel educativo obtenido determina hasta cierto punto los recursos monetarios y el reconocimiento social disponible para los individuos en sus vidas subsiguientes, debido a que la educación es un poderoso factor - y se ha vuelto así cada vez más en el transcurso del siglo
XIX y XX - que permite o impide el acceso a ocupaciones de privilegio y provee los medios para los logros profesionales $y$, por ende, de remuneración y status socioeconómico. Estas funciones básicas son indiscutibles (Lobo y Morua, 2011, p. 55).

Harald Schomburg (2004) considera que en un análisis de relación entre educación superior y el "mundo del trabajo" se debe basar en los siguientes enfoques:

- Una mirada a los temas del mercado de empleo, es decir datos cuantitativos y estructurales sobre empleo y carreras.

- Un enfoque más comprometido con el carácter del trabajo y las competencias relacionadas.

- Una evaluación directa de las condiciones de estudio y los recursos de acuerdo a la experiencia de los graduados,

- Una comprensión de los valores y las orientaciones de los graduados, y finalmente;

- La recolección de información, con enfoque retrospectivo, de la educación superior (p. 40).

En la entrevista telefónica a Mario Morua (2012), los estudios en relación con el "mundo del trabajo" pueden tener su aplicación en varios momentos, o según el objetivo de la investigación, tal como se aprecia en Lobo y Morua (2011, p. 64):

- Al momento de ingresar al programa de postgrado

- Durante los estudios

- Al momento de graduarse

- Al momento de realizar la encuesta

Al respecto, Morua considera que los temas por tratar en cada uno de esos momentos depen- 
den del contexto en que se desarrolla el estudio y de los objetivos o fines que se persiguen con la investigación. Para Harald Schomburg (2004) la relevancia práctica de este tipo de estudio de seguimiento de relación entre el "mundo de trabajo" y la universidad permite plantear una serie de temas con el fin de conocer la experiencia en:

- Situación de empleo: salario, sector económico, títulos de los empleos, jornada de trabajo, duración de la búsqueda del primer empleo y métodos de búsqueda de trabajo, entre otros.

- Cooperación y contactos: los estudios de relación permiten establecer relaciones de cooperación y contacto entre las universidades, los alumnos, egresado, y el mercado laboral.

- Tareas laborales y competencias requeridas: los tipos de estudios de relación mercado laboral y universidad no deben restringirse a mediciones típicas al mercado de trabajo como sector económico, grupos de ocupación, estatus, y salarios. La información sobre condiciones de ocupación y el tipo de competencias laborales requeridas son una fuente más rica para la innovación curricular que solo los datos del mercado de trabajo.

- Currículo y preparación del trabajo: se refieren al conocimiento práctico, abordaje de problemas, tipos de aprendizaje.

- Entre otros temas como datos de entrada del estudiante, características del empleador, y otros que se abordan de acuerdo al interés propio de la investigación y del postgrado del estudiante (pp. 39-44).

Por la experiencia señalada en estos tipos de estudio, en cuanto al "mundo del trabajo" y la universidad, Harald Schomburg (2004) destaca que para el Centro para la Investigación en Educación Superior y Trabajo de la Universidad de Kassel en Alemania, los estudios sobre gradua- dos han sido uno de los principales campos de investigación, entre los cuales se referencia de manera textual:

- Estudios secundarios referentes a las consecuencias de la expansión universitaria con respecto a la relación entre educación superior y trabajo.

- Estudios secundarios en relación al estatus de los estudios de graduados para la reestructuración de los programas de estudio. Estos se realizaron para identificar posibles formas de relacionar las actividades profesionales de los graduados con los requerimientos y potencialidades de los programas de estudio y enseñanza (Holtkamp/Teichler, 1983).

- Los estudios anteriores fueron enriquecidos por un estudio realizado con administradores de personal sobre la variedad (subestimada) de criterios y métodos de reclutamiento de graduados universitarios (Teichler/ Buttgereit/ Holtkamp, 1984).

- Un estudio longitudinal de graduados con casi una década de duración (a los dos años, cinco años y diez años después de la graduación) ha sido completado con la intención de mostrar, más claramente, la complejidad de las opciones y condiciones de un programa de estudio y su importancia para el desarrollo profesional de los graduados universitarios (Teichler/ Schomburg/Winkler, 1992).

- El estudio Transición de la Educación Superior al Mercado Laboral en Japón fue desarrollado a inicios de los 90 . Sus métodos principales fueron entrevistas en grandes empresas japonesas relacionadas con los criterios y métodos de investigación para el reclutamiento de graduados universitarios, así como la conexión entre las competencias profesionales esperadas en el momento de entrada al mercado laboral, las competencias inicia- 
les reales y la carrera profesional posterior de los graduados (p. 9).

En Lobo y Morua (2011), se resume que con la aplicación de dichos aportes en estudios llevados en la Universidad de Kassel, Universidad Libre de Berlín y Universidad Humboldt, los logros han sido los siguientes:

- Han identificado con certeza y precisión lo que el mercado laboral requiere en términos de formación del factor humano a nivel de educación superior.

- En esa misma línea de investigación, los estudios han sido utilizados para monitorear el impacto que sus graduados están teniendo profesionalmente en el mercado laboral nacional como internacional, como mejorar la calidad académica en los programas que así lo requieren, siempre en función de ofrecer una respuesta asertiva al mercado laboral (p. 53).

En consideración de los aportes de ambos profesores de la Universidad de Kassel, según Lobo y Morua (2011) los estudios de seguimiento a personas graduadas de posgrados desarrollados en Costa Rica, se constituyen en un instrumento que permite monitorear, verificar y validar las tres funciones planteadas por Schomburg en relación con el "mundo del trabajo"y las universidades.

A partir del marco referencial y las experiencias citadas sobre los estudios relacionados con el "mundo del trabajo", se considera que las contribuciones de Schomburg (2004) y Teichler (2005) exponen una base teórica aplicable (funciones relacionadas, enfoques de estudio, momentos aplicativos, temas de estudios) que vincula y sustenta la importancia de llevar a cabo estudios sobre aspectos relacionados con el mercado laboral de personas que estudian y trabajan a la vez, tal como se plantea en el apartado metodológico de la presente investigación.
La educación que recibe el estudiante de maestría es una forma de prepararse para enfrentar el trabajo actual o futuro. Es por esto que la información sobre los elementos del mercado laboral relacionado con estudiantes de maestría de Negocios de ULACIT, se considera un tema importante de estudio.

\section{Metodología Tipo de estudio}

La investigación se desarrolló mediante una metodología descriptiva, es decir, se observa, sin manipular las variables. Los datos recolectados para el periodo dado son descriptivos porque son analizados y discutidos tal como son.

\section{Participantes}

Para llevar a cabo el presente artículo se configuró una muestra con 50 estudiantes de maestría de Negocios de la Universidad Latinoamericana de Ciencia y Tecnología (ULACIT) que han trabajado y estudiado durante el año 2012. La muestra está constituida por 29 hombres (58\%) y 21 mujeres (42\%), cuyas edades van desde los 22 años hasta los 54 años.

Se ha seleccionado la muestra por conveniencia, debido a la disponibilidad de los estudiantes para poder responder el instrumento de recolección de datos dentro de las instalaciones de la universidad. Por otro lado, ha sido en la ULACIT donde algunos estudiantes de la maestría de Negocios han expresado directamente al investigador la necesidad de conocer más sobre el mercado laboral en que se desarrollan profesionalmente.

La cantidad de estudiantes que forman la muestra en estudio se acoge al número propuesto por los miembros representantes de la universidad para los cursos de investigación aplicada que ofrece la ULACIT en el Sistema de Posgrado. 


\section{Instrumentos}

Para la recolección de los datos se utilizó un cuestionario que fue estructurado en cinco partes y catorce interrogantes: aspectos generales del encuestado (cuatro opciones), características y condiciones de empleo (cinco preguntas), obtención de empleo (dos preguntas), ingresos (una pregunta), formación (una pregunta). La mayoría de las preguntas son de respuesta múltiple.

Como técnicas alternas de obtención de información, se utiliza básicamente la revisión documental y bibliográfica como insumo al desarrollo del proceso o planteamiento de la investigación.

Asimismo, se consultó vía telefónica a personas que laboran en posiciones de recursos humanos, con el fin de obtener su punto de vista para el diseño del instrumento aplicado en la investigación. La técnica de observación siempre estuvo ligada a apreciaciones y discusiones que se mantuvieron presentes durante la investigación.

\section{Procedimientos}

Para elaborar esta investigación se siguió un enfoque mixto, que implica la recolección y el análisis de datos cuantitativos y cualitativos que permitan lograr con mayor entendimiento del tema en estudio (Tabla 1).

Previo a la aplicación del instrumento de recolección de datos, se realizó un pre-testeo cognitivo con el fin de detectar ambigüedades y problemas de interpretación de las preguntas, tiempo de duración, claridad de la redacción y congruencia del cuestionario en general. El análisis se llevo a cabo con diez personas que no presentan relación alguna con la muestra por investigar, lo cual incrementó la posibilidad de interpretación adecuada de la encuesta.

Una vez obtenidos los datos, se procedió a la respectiva codificación y tabulación con el propósito organizar la información.

\section{Resultados}

Según la muestra utilizada en el presente estudio, el interés de los estudiantes por el mundo de los negocios se diversifica a partir de la elección del énfasis académico por el cual optan. En ese sentido, 50\% de los estudiantes encuestados durante la investigación estudiaban Gerencia de Producción y Operaciones, mientras que los estudiantes en Finanzas representaban 20\% y de Mercadeo 16\%. Otros estudiantes preferían estudiar Recursos Humanos, Comercio o Gerencia de Calidad.

Figura 1

ÉNFASIS DEL MBA QUE LAS PERSONAS PREFIEREN ESTUDIAR EN ULACIT

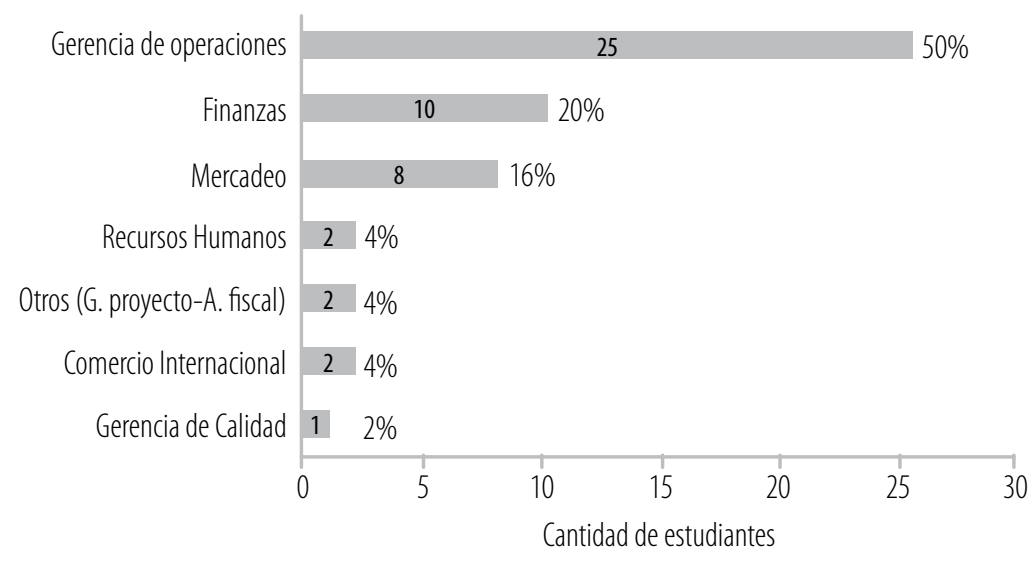

Fuente: Elaboración propia. 
TABLA 1

\section{MATRIZ BÁSICA DE INVESTIGACIÓN}

\section{Objetivo general de investigación}

Identificar los principales aspectos relacionados con el mercado laboral de estudiantes de maestría de la carrera de Negocios de ULACIT, quienes simultáneamente han trabajado y estudiado durante el 2012, con respecto a oportunidades de empleo, actividades, formas para buscar empleo, métodos de selección de empleados, ingresos y áreas claves de formación requeridas en el desarrollo profesional.

\begin{tabular}{|c|c|c|}
\hline Objetivo específico & Variable & Indicador \\
\hline $\begin{array}{l}\text { Identificar el tipo de empresa donde } \\
\text { un estudiante de maestría en negocios } \\
\text { tiene oportunidad de conseguir empleo. }\end{array}$ & $\begin{array}{l}\text { Oportunidad } \\
\text { de empleo }\end{array}$ & $\begin{array}{l}\text { A) Características y condiciones de empleo: } \\
\text { 1. Posesión de empleo } \\
\text { 2. Cantidad de empleo } \\
\text { 3. Ejercicio de la carrera } \\
\text { 4. Lugar de empleo }\end{array}$ \\
\hline $\begin{array}{l}\text { Determinar los principales cargos y } \\
\text { actividades que efectúan los estudiantes } \\
\text { de maestría en negocios. }\end{array}$ & $\begin{array}{c}\text { Cargos } \\
\text { y actividades }\end{array}$ & $\begin{array}{l}\text { 1. Tipos de cargos } \\
\text { 2. Tipos de actividades que realizan: operaciones generales de negocios, cadena de } \\
\text { suministro, cadena de valor, logística, fabricación, mercadeo, ventas, servicio al cliente, } \\
\text { financiero, recursos humanos, I+ D, TI, calidad. } \\
\text { 3. Razones que lo instan a realizar sus actividades: salario, crecimiento profesional, sa- } \\
\text { tisfacción personal, beneficios laborales (médico de empresa, seguro familiar privado, } \\
\text { beca de estudio), sin opciones laborales. }\end{array}$ \\
\hline $\begin{array}{l}\text { Determinar los principales medios que } \\
\text { los estudiantes de maestría de negocios } \\
\text { utilizan para buscar y conseguir empleo. }\end{array}$ & $\begin{array}{l}\text { Medio de búsqueda } \\
\text { de empleo }\end{array}$ & $\begin{array}{l}\text { B) Obtención de empleo } \\
\text { 1. Aspectos que le permiten participar en el mercado laboral: la maestría en nego- } \\
\text { cios, contar con experiencia previa, la experiencia obtenida en el actual empleo, } \\
\text { contar con habilidades idiomáticas, otros como (SAP, vehículo propio, liderazgo, } \\
\text { trabajo en equipo, incorporación al colegio respectivo). } \\
\text { 2. Formas de conseguir empleo: redes de contactos personales (familiares y amigos), } \\
\text { redes de contacto profesionales (profesores, compañeros), iniciar una idea propia } \\
\text { de negocio, redes de internet, bolsas de empleo, periódicos. }\end{array}$ \\
\hline $\begin{array}{l}\text { Conocer por medio del encuestado los } \\
\text { métodos que las empresas utilizan para } \\
\text { la selección de estudiantes de maestría } \\
\text { en negocios. }\end{array}$ & $\begin{array}{c}\text { Métodos de selección } \\
\text { de personal }\end{array}$ & $\begin{array}{l}\text { 1. Formas en que las empresas seleccionan estudiantes de maestría en negocios: } \\
\text { revisión del curriculum (edad, experiencias, competencias, expectativas salarial, } \\
\text { otros), entrevista, simulación de un día de trabajo, pruebas de conocimiento, } \\
\text { cartas de recomendación, métodos de reclutamiento en línea. }\end{array}$ \\
\hline $\begin{array}{l}\text { Dar a conocer las opiniones de los } \\
\text { estudiantes de maestría en negocios } \\
\text { respecto a los ingresos y a las áreas clave } \\
\text { de formación. }\end{array}$ & Ingreso / Formación & $\begin{array}{l}\text { C) Ingresos } \\
\text { 1. Rango salarial de estudiantes de maestría en negocios. } \\
\text { 2. Factores que permiten recibir esos ingresos: estudios de postgrados, calidad } \\
\text { académica de la universidad en que estudia, experiencia laboral, dominio del } \\
\text { idioma Inglés, otro. } \\
\text { Formación } \\
\text { 3. Áreas clave de formación para el 2013: dirección, cuadros de mando integral, al- } \\
\text { fabetización tecnológica, entrenamiento en habilidad (liderazgo, cultura, gestión } \\
\text { de cambio), certificarse en algún tipo de proceso, ser trilingüe (inglés, español, } \\
\text { mandarín), otros. }\end{array}$ \\
\hline
\end{tabular}

Fuevte: Elaboración propia. 


\section{Características y condiciones de empleo}

Oportunidad de empleo

Se debe resaltar que las 50 personas encuestadas poseen empleo, es decir, la tasa de ocupación es de 100\%, lo cual se puede relacionar más adelante con la experiencia, el liderazgo y el trabajo en equipo que poseen los estudiantes.

Por la cantidad de relaciones laborales que presentan los estudiantes en el mercado laboral costarricense, se muestra la oportunidad de cambio de un trabajo a otro. Según los datos obtenidos, $30 \%$ ha contado con tres empleos y $14 \%$ más de cinco empleos en toda su vida laboral.

La rotación de empleo que proviene de las personas que han tenido más de tres empleos (78\%), no solo demuestra la posibilidad de encontrar un lugar donde trabajar, sino el tener un lugar de trabajo que le permita crecimiento, mayores expectativas salariales y satisfacción personal.

\section{FiguRA 2}

\section{¿CUÁNTOS EMPLEOS HA TENIDO?}

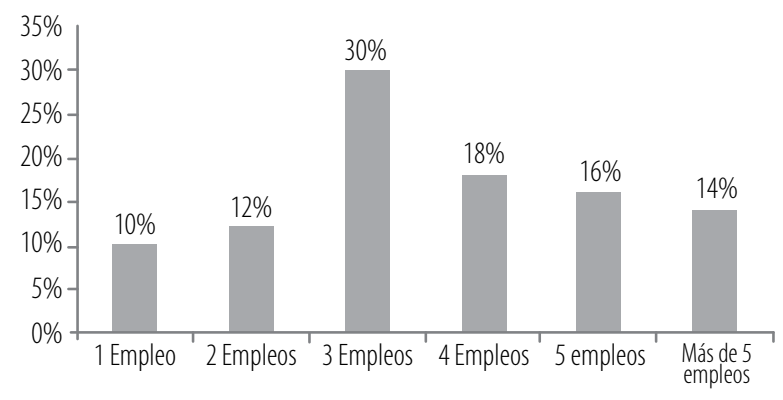

Fuente: Elaboración propia.

Con respecto al ejercicio de la carrera, $88 \%$ de los estudiantes expresa que ha ejecutado actividades relacionadas con su postgrado, $82 \%$ en su empleo actual y $6 \%$ en empleos anteriores. Esto significa que solo durante el 2012, 18\% de los encuestados corresponde a personas cuyo trabajo actual no está relacionado a sus estudios, lo cual quiere decir que $12 \%$ nunca ha tenido un empleo ligado a su profesión.

\section{FIGURA 3}

\section{¿HA TENIDO EMPLEOS RELACIONADOS CON SU CARRERA?}

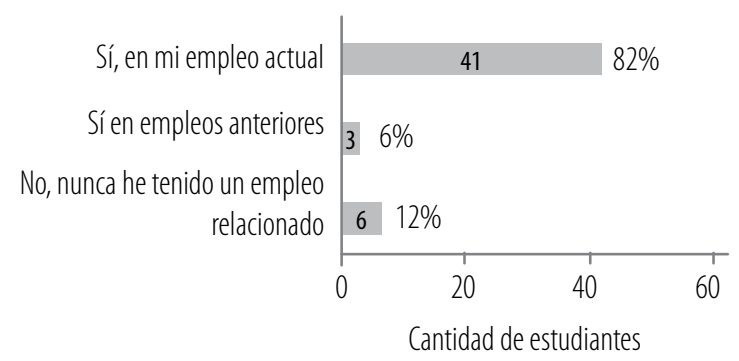

Fuente: Elaboración propia.

La experiencia de la mayoría de los estudiantes de maestría en negocios al encontrar empleo relacionado a sus carreras de estudio permite, les ha facilitado construir una vida profesional más exitosa y llena de satisfacción. Los lugares de empleo donde los estudiantes de maestría se desarrollan profesionalmente son las organizaciones privadas y públicas que representan 92\% y $8 \%$, respectivamente.

\section{Cargos y actividades}

Mediante los resultados obtenidos se les exponen a los actuales y futuros estudiantes aquellos cargos y actividades que son desempeñados especialmente en las empresas privadas, lo anterior con el fin de que estos aspectos del mercado laboral costarricense le puedan orientar el conocimiento de la muestra en estudio sobre la especialidad que exigen las empresas generadoras de empleo.

Así, 34\% de los estudiantes de maestría de Negocios de ULACIT se desempeña especialmente en cargos gerenciales tales como: gerentes financieros, gerentes contables, gerentes de auditorías, gerente de operaciones, gerente de producción, gerentes de tecnologías, gerentes de mercadeo, gerentes de proyectos, gerentes de logística, gerentes de almacén, también se reportó un director y un vicepresidente empresarial. 
Por otro lado, en un porcentaje igual de 14\%, los estudiantes encuestados ocupan posiciones de servicios y consultorías, administradores y jefaturas. Mientras las posiciones de ingenieros y analistas son ocupadas por $12 \%$ de los alumnos respectivamente.

Un dato interesante extraído de la encuesta es que las posiciones gerenciales están lideradas por mujeres, especialmente en las gerencias financieras, gerencias contables y gerencias de auditorías.

\section{FIgURA 4}

\section{CARGO ACTUAL DE OCUPACIÓN}

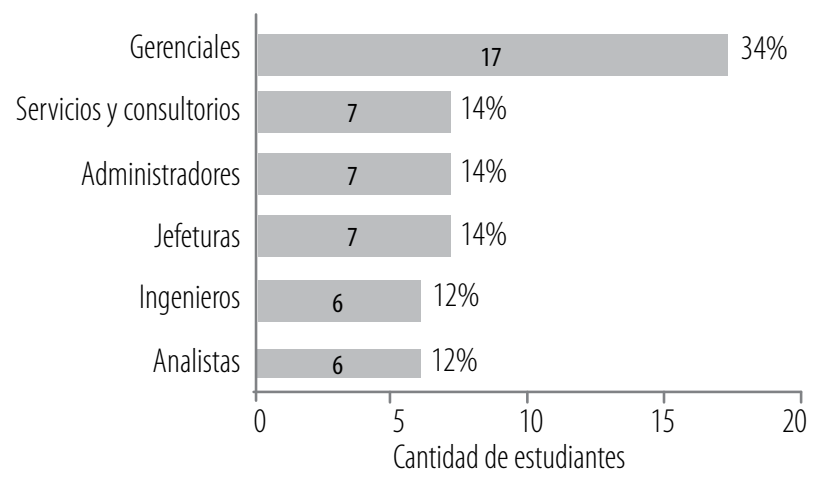

Fuente: Elaboración propia.

A partir de la experiencia de los estudiantes en sus cargos, se determinaron las principales actividades que siempre u ocasionalmente realizan en sus puestos de trabajo.

Como respuesta múltiple, 54\% de los estudiantes manifestó que en su trabajo siempre realizan actividades de logística, seguido de operaciones generales de negocios 52\%; por otra parte, $46 \%$ lleva a cabo actividades de servicio al cliente, mientras 36\% lleva a cabo actividades en finanzas.

La alta participación de los estudiantes en las actividades mencionadas hace pensar que las cifras mantienen una relación con el énfasis de especialidades por el cual los estudiantes optan en la universidad (FIGURA 1) y la alta rotación de empleo por parte de aquellas personas que ha tenido más de tres y cinco empleos.

Otras actividades de vital importancia son aquellas relacionadas con calidad, venta, cadenas de suministros, cadena de valor, recursos humanos, mercadeo, tecnología e información, producción, investigación y desarrollo, siendo esta última una actividad que solamente $8 \%$ de los encuestados desarrollan en su cargos de trabajo (FIGURA 5).

Al observar la figura 5, se detecta que casi de manera inversa las actividades que no siempre se hacen son las que ocasionalmente llevan a cabo la mayoría de los estudiantes. Es decir, 30\% de la muestra ocasionalmente ejecuta actividades de mercadeo, $28 \%$ en ventas y $24 \%$ en $\mathrm{Tl}$ e $I+D$, respectivamente. Lo cual puede indicarnos que la mayoría de los estudiantes (FIGURA 3) tiene un empleo relacionado con su carrera.

Independientemente de la organización en que el estudiante trabaje, sea esta pública o privada, es también necesario indicar las razones por la cuales se encuentran trabajando en la actual empresa. Como respuesta múltiple, 68\% de los estudiantes encuestados manifiesta estar trabajando en la actual empresa por un asunto de crecimiento profesional. El salario es la segunda razón mencionada con 46\%, seguido de la satisfacción personal con 38\%, así como 18\% indica gozar de beneficios como médicos de empresa, seguro familiar y becas de estudio. Otro 18\% sorprende al indicar que trabaja en la empresa porque no tenía más opciones laborales.

\section{Obtención de empleo Búsqueda de empleo}

Cuando se piensa en búsqueda de empleo dentro del mercado laboral costarricense se deben contemplar dos variables: los aspectos que permiten participar activamente en el mercado laboral y la forma de conseguir el empleo. 


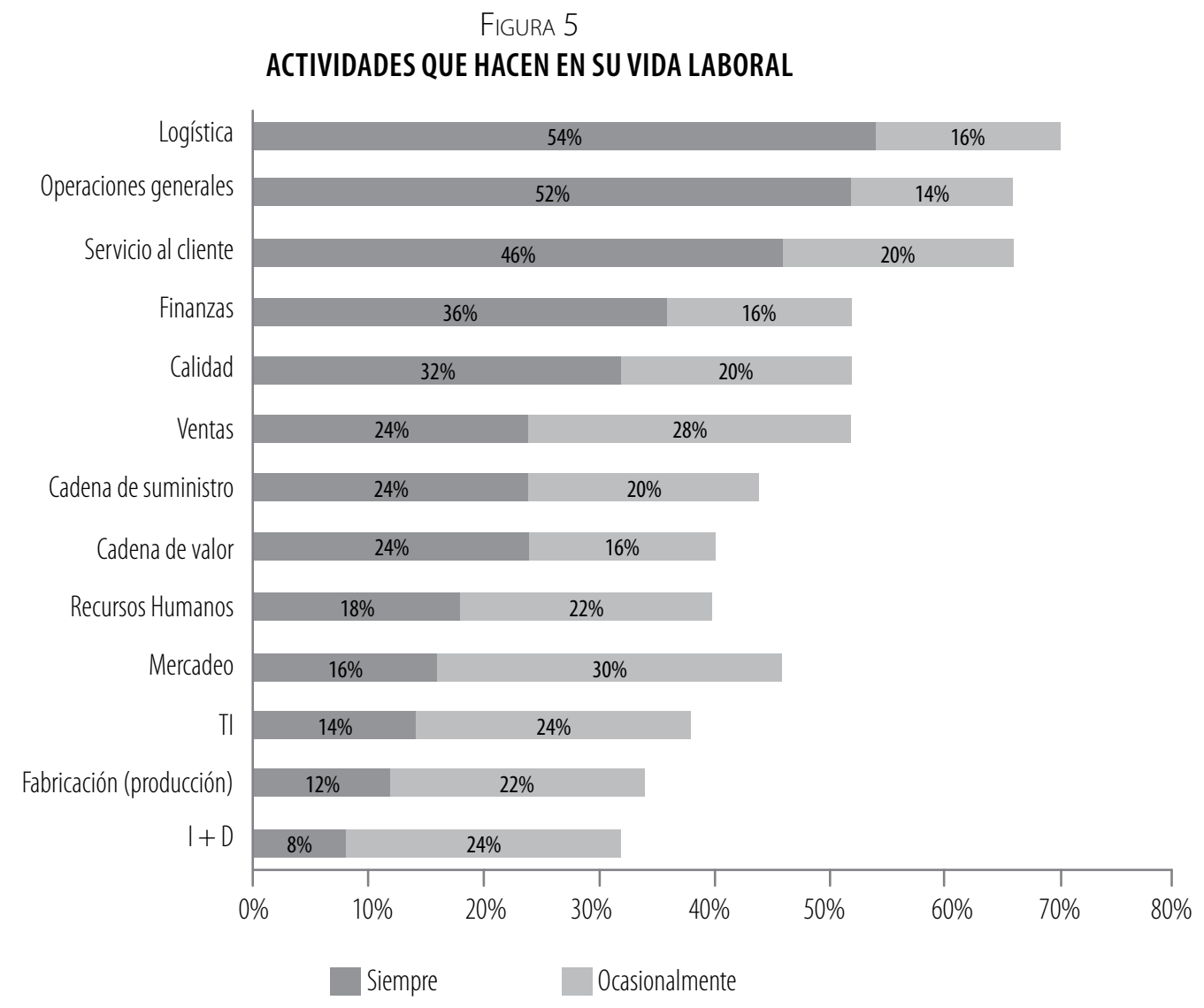

Fuente: Elaboración propia.

FIGURA 6

RAZONES MÁS IMPORTANTES POR LAS CUALES SE ENCUENTRAN TRABAJANDO EN LA ACTUAL EMPRESA

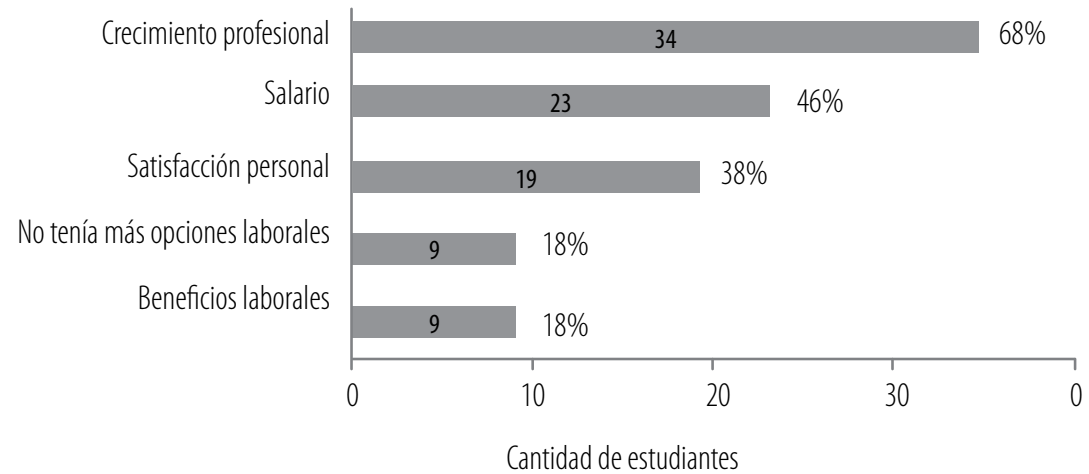

Fuente: Elaboración propia. 
En cuanto a las múltiples respuestas, 58\% de los encuestados consideran que los aspectos más importantes que han mediado para la participación en el mercado laboral y realizar las actividades propias de su puesto de trabajo son aquellas especialmente relacionadas con la experiencia previa que ha adquirido en otros lugares de trabajo en puestos iguales o similares al actual.

El liderazgo y el trabajo en equipo ocupa el segundo lugar con 54\%, el cual se puede ver fortalecido con la experiencia que han acumulado las personas en sus anteriores trabajos y la sumativa del trabajo actual que representa 34\% y que constituye el interés y el compromiso con sus deberes.

Según 26\% de los encuestados, las habilidades idiomáticas son un aspecto de interés para los puestos de trabajo; sin embargo, este ya no representa un plus sino que simplemente se debe contar con idiomas tales como el inglés, el mandarín y el portugués.
Otros aspectos como el haber iniciado una maestría en Negocios, tener conocimientos en SAP (Sistema, Aplicaciones y Productos) o estar incorporado al colegio respectivo, no muestran mayor relevancia para ingresar al mercado laboral. La percepción sobre este punto es que si las empresas a futuro requieren a alguien con este curriculum simplemente los capacitan o los benefician con becas de estudio y así también se crea fidelidad para con la organización.

Los estudiantes de maestría de ULACIT han utilizado diferentes métodos de búsqueda de empleo, entre ellos las bolsas de empleos han sido la mejor estrategia para tales efectos, según lo ha manifestado 30\% de los encuestados.

Por otra parte, las redes de contactos profesionales y redes de contacto personal son opciones que utilizaron para obtener su actual empleo. Es decir, 20\% consiguió su empleo a través de profesores y compañeros, mientras que otro $20 \%$ se apoyó en familiares y amigos.

FIGURA 7

\section{ASPECTOS DE INSERCIÓN QUE LE HAN PERMITIDO REALIZAR LAS ACTIVIDADES LABORALES}

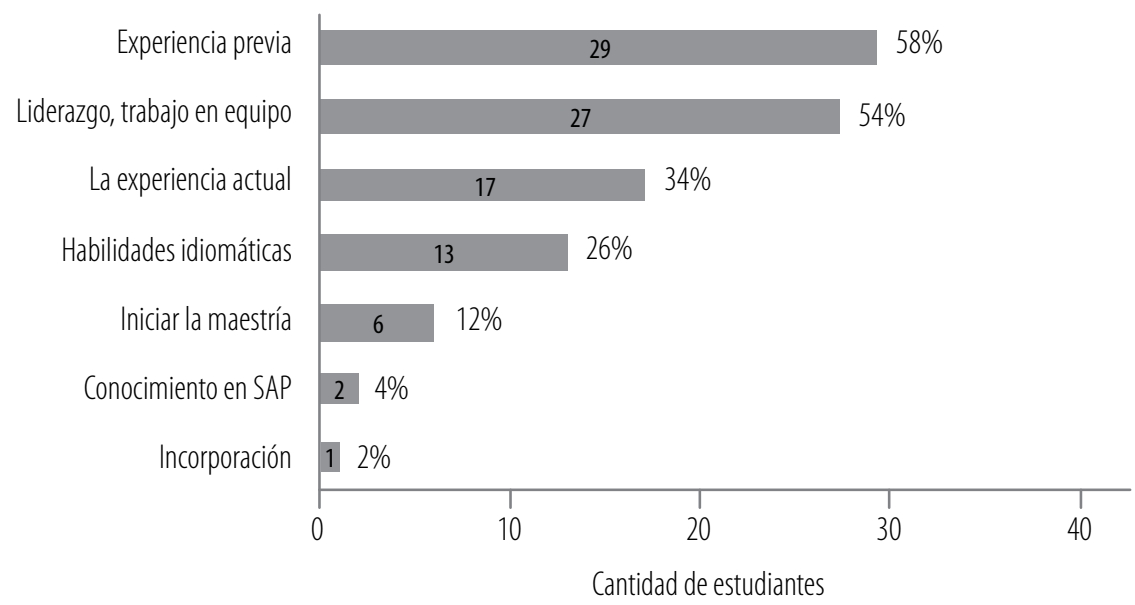

Fuente: Elaboración propia. 
Para 12\% de los informantes, las ferias de empleo y la idea de tener un negocio propio fueron el vehículo para la obtención de su trabajo actual, seguido de $8 \%$ que lo logró por medio de un ascenso. Por otra parte, $6 \%$ de los estudiantes adquirió su trabajo por medio del periódico y 4\% utilizó las redes de Internet para obtener su último empleo.

\section{Métodos de selección de personal}

A lo largo de la carrera laboral, los estudiantes encuestados han tenido en promedio tres empleos y han sido siete los medios de búsqueda utilizados para obtener su último trabajo. Lo anterior nos permitió indagar sobre los métodos que las empresas utilizan para seleccionarlos como empleados en sus organizaciones.

FIGURA 8

\section{¿DE QUÉ FORMA ADQUIRIÓ SU ACTUAL EMPLEO?}

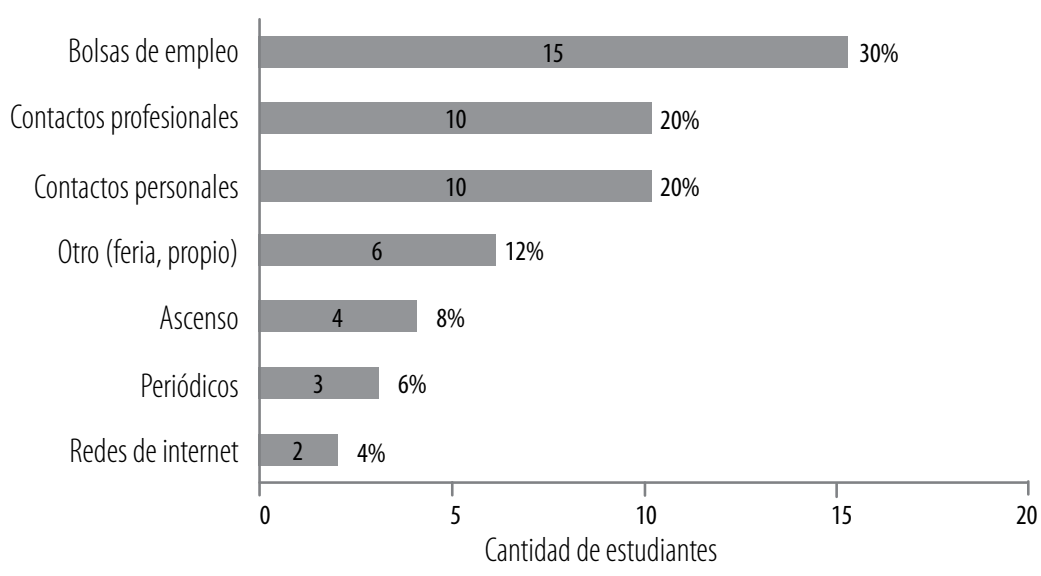

Fuente: Elaboración propia.

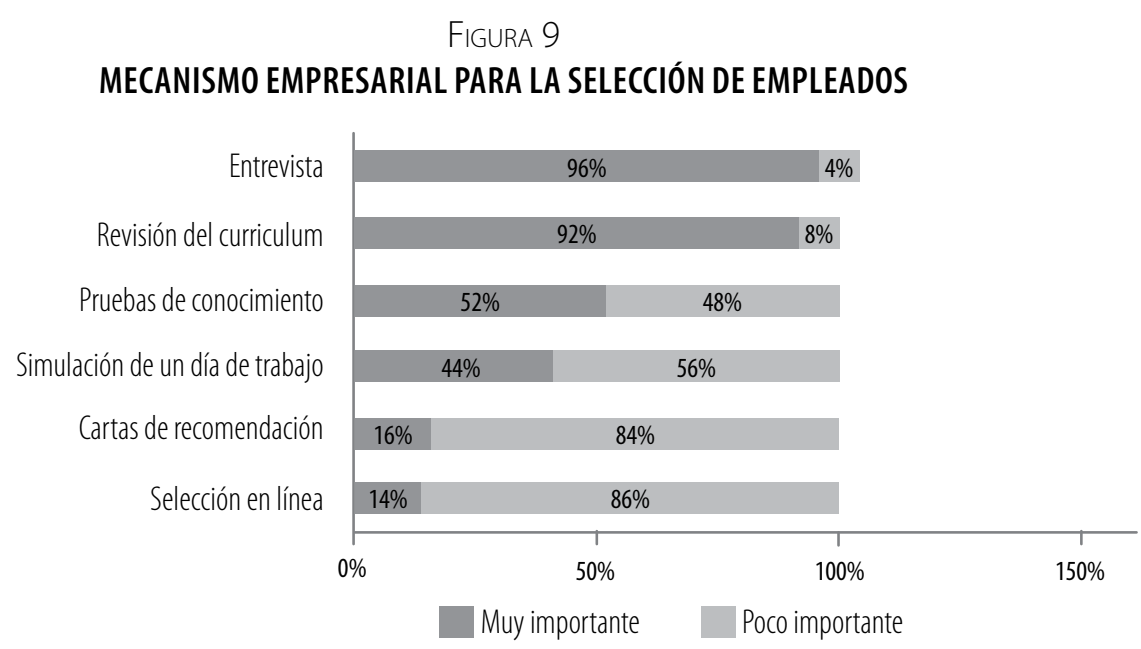

Fuente: Elaboración propia. 
La entrevista y la revisión del curriculum son los mecanismos más importantes que las empresas utilizan para la selección de sus empleados según opina respectivamente $96 \%$ y $92 \%$ de los encuestados. Sin embargo, en ese mismo orden, existe una minoría de $4 \%$ y $8 \%$ para quienes son poco importantes los mecanismos citados.

Para 52\% de los estudiantes es muy importante que las empresas apliquen pruebas de conocimiento en algún momento del proceso de reclutamiento mientras que el resto lo considera poco importante. Paralelo a este mecanismo solo 48\% considera relevante un día de simulación de trabajo, por debajo del 56\% para quienes este punto no es importante.

Se observa que $84 \%$ y $86 \%$ de los estudiantes considera -respectivamente- que las cartas de recomendación y la selección en línea son aspectos poco importantes dentro del proceso de selección de las empresas.

\section{Ingresos}

Se debe resaltar que el ingreso es un tema muy sensible para los encuestados, por lo cual se definieron rangos de salarios. Sin embargo, pudo existir algún motivo para no responder con sinceridad a esta pregunta.
El ingreso salarial más común que ganan los estudiantes de maestría de Negocios de ULACIT se concentra entre el rango US\$1501- US\$ 2000 mensuales según 22\% de los encuestados, mientras que $20 \%$ de dicha muestra gana entre US\$2001-US\$ 2500 al mes.

Se observa que $12 \%$ de los estudiantes gana entre US\$1000-US\$ 1500 al mes; 8\% entre US\$ 3001US\$ 3500 mensuales, mientras que en un porcentaje igual un grupo de estudiantes gana menos de US\$ 1000 al mes, el salario más bajo registrado en la encuesta, en contraste con una minoría que gana por encima de US\$3000 y US\$ 5000 al mes.

A la vista de estas cifras, la escala de salarios es diversa como lo son los factores que permiten recibir esos ingresos, es decir, 86\% de los encuestados consideran que el principal factor para obtener su salario ha sido la experiencia laboral, lo cual se relaciona también con los aspectos que les permitieron ingresar al mercado laboral (FIGURA 7).

Los demás elementos como contar con una maestría en negocios, el ser empresario, la calidad académica recibida de la universidad, el inglés, son igualmente condiciones que a otros estudiantes les ha facultado para mantener un rango salarial aceptable al mes.

\section{FIGURA 10 \\ RANGO DE LOS ESTUDIANTES DE MAESTRÍA DE NEGOCIOS DE ULACIT}

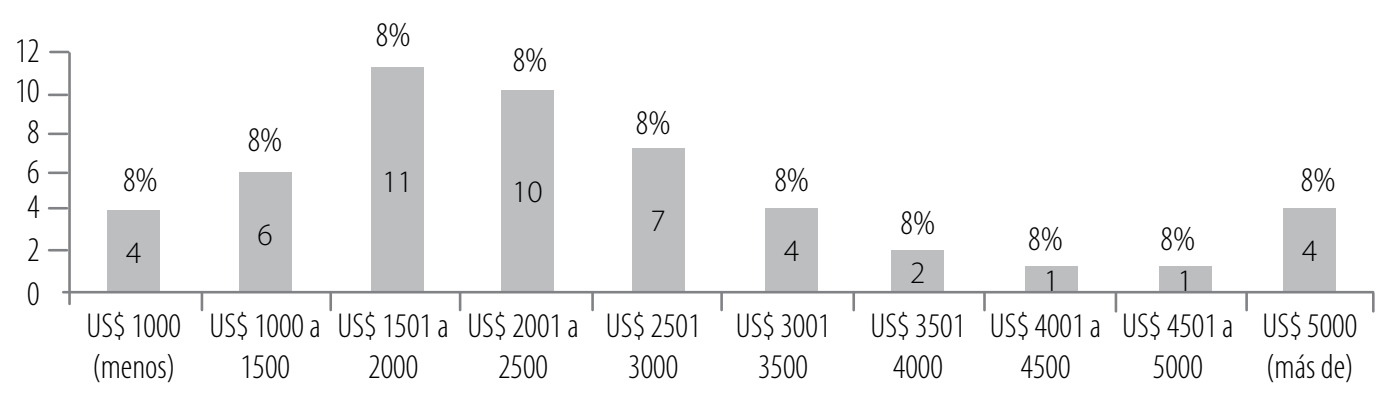

Fuente: Elaboración propia. 
FIGURA 11

\section{FACTORES QUE PERMITEN RECIBIR MAYORES INGRESOS}

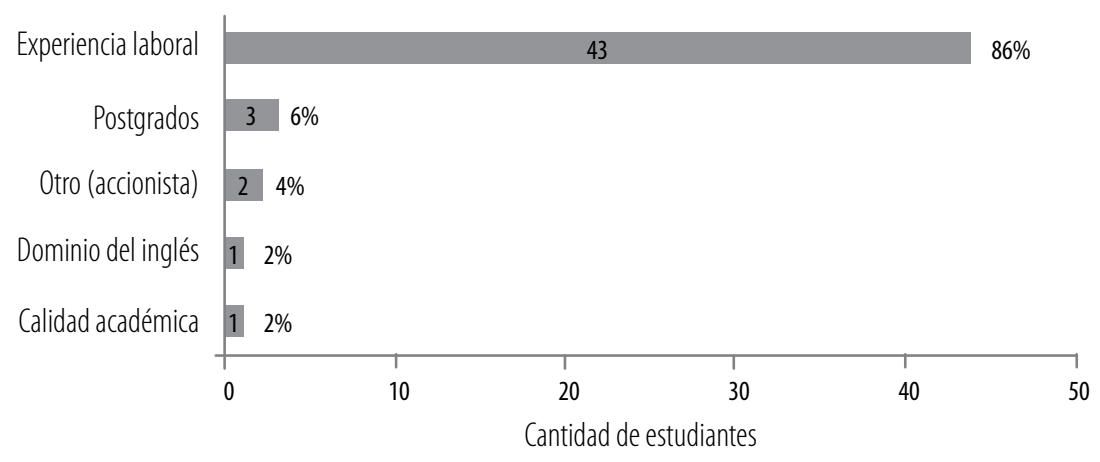

Fuente: Elaboración propia.

\section{Formación}

En la encuesta aplicada a la muestra se indagó sobre cuáles áreas de conocimiento se consideran claves en la formación profesional para el 2013, las cuales permiten seguir dentro del mercado laboral. En este contexto, en las respuestas múltiples los siguientes fueron los resultados obtenidos:

Para 64\% la formación profesional para el año 2013 debe estar orientada al liderazgo, cambios de gestión, y habilidades técnicas que les permitan un mejor desarrollo laboral.

De los encuestados $42 \%$ siente la necesidad de certificarse en algún tipo de proceso. Podemos inferir que este dato está relacionado con 25\% de los estudiantes que opta por el título de Gerencia de Operaciones, en cuyo curso de de Producción y Operaciones uno de los objetivos es que el estudiante comprenda la importancia de especializarse en algún proceso que le permita mayores competencias frente a los demás dentro del mercado laboral.
Idiomas como el inglés, el mandarín y el portugués cobran su importancia para 36\% de los estudiantes como una las áreas prioritarias de formación para el futuro. Esto a pesar de que no es un factor que les permite recibir mayores ingresos pero sí para insertarse en el mercado laboral y poder llevar a cabo óptimamente las actividades propias de su cargo.

A pesar de que los estudiantes están dentro de un programa de maestría, 32\% considera que los programas de dirección fuera de campo de la maestría son de vital importancia para el contexto laboral en que se desarrollan profesionalmente.

El 26\% y 22\% de los estudiantes de maestría considera que las capacitaciones en cuadro de mandos integrales y la consecución de programas de alfabetización tecnológica respectivamente, son sus prioridades de formación para el año 2013. 


\section{ÁREAS DE FORMACÓN PROFESIONAL CLAVES PARA EL AÑO 2013}

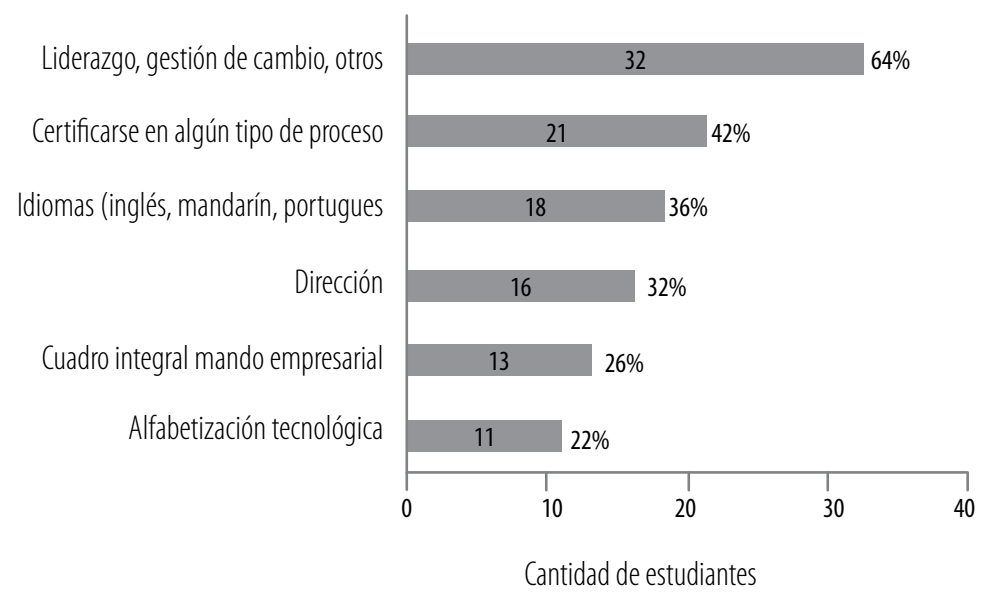

Fuente: Elaboración propia.

\section{Conclusiones}

A la hora de integrar los datos de la investigación, se concluye que las organizaciones generadoras de empleo, las actividades que los estudiantes realizan en sus cargos, las formas de buscar empleo por parte de los estudiantes, los métodos de selección de personal utilizados por las empresa, los ingresos y las formaciones futuras, son los principales factores del mercado laboral que los estudiantes de maestría de Negocios de la muestra en estudio deben tener en cuenta cuando deciden ingresar en el mercado laboral costarricense. Estar al tanto de los factores citados les permitirá conocer lo que les espera en el mercado laboral durante su carrera profesional y después de graduarse.

- Oportunidades de empleo: Los 50 estudiantes de maestría de Negocios de ULACIT que conformaron la muestra poseían trabajo al momento de la encuesta. Las organizaciones del sector privado representan
92\%. Así bien, los estudiantes de maestría de Negocios tienen una oportunidad de encontrar empleo y desarrollarse profesionalmente en el mercado laboral. Por la cantidad de empleos que los estudiantes han tenido en su vida laboral, se muestra la oportunidad de cambio de un trabajo a otro. Sin embargo, $18 \%$ de los estudiantes durante el 2012 poseían empleo pero no relacionado con su carrera de estudio. El $78 \%$ de la rotación no solo indica la oportunidad de encontrar un lugar de trabajo, sino que representa la opción de tener un trabajo que le permita crecimiento, mayores expectativas salariales y crecimiento personal.

- Cargos y actividades: El mercado laboral le ha permitido a 34\% de los estudiantes de maestría encuestados desarrollarse especialmente en cargos gerenciales en los que han podido llevar a cabo especialmente actividades de logística, ope- 
raciones generales del negocio, servicio al cliente y finanzas. Un dato interesante de la investigación es que las posiciones gerenciales está liderada por mujeres en $20 \%$, especialmente en las gerencias financieras, gerencias contables y gerencias de auditorías. Algunas razones que motivan en el desempeño son especialmente el crecimiento profesional (68\%), el salario (46\%) y la satisfacción personal (38\%).

- Medio de búsqueda de empleo: Lo que el estudiante ofrezca al empleador y el medio que utilice para conseguir empleo son dos aspectos importantes de entrada a la hora de buscar trabajo. En ese sentido, la experiencia es el factor más importante para participar y tener éxito en el mercado laboral costarricense (58\%), seguido del liderazgo y el trabajo en equipo (34\%). Las bolsas de empleo son el medio que $30 \%$ de los estudiantes utiliza para buscar un empleo, seguido de los contactos profesionales (20\%) y personales (20\%).

- Método de selección de personal: Como respuesta múltiple sobre los mecanismos que las empresas utilizan para la selección del personal, los estudiantes de maestría en estudio consideran como muy importante las tradicionales entrevistas (96\%) y la revisión del curriculum (92\%), y otras como las pruebas de conocimiento (52\%) y la simulación de trabajo (44\%).
- Ingresos y formación: El ingreso común de los estudiantes de maestría de Negocios se observó en el rango de US\$1501-US\$ 2000. Los bajos salarios son menos de US\$ 1000 mensuales en comparación al salario más alto que supera los US\$5000 mensuales. Las diferencias entre lo que gana una persona de otra está orientado en los niveles de experiencia laboral con que cuentan los estudiantes y no por su carrera universitaria a nivel de posgrado.

\section{Recomendaciones}

Oportunidades de empleo: Debido a que la estructura pública apenas representa $8 \%$ de la oportunidad laboral para la muestra en estudio, los estudiantes deben buscar espacios de contactos y aprovechar posiciones de trabajo que el gobierno genera por medio de la inversión extranjera directa, cada vez es más exigente a la hora de buscar el talento humano para sus estructuras.

- Cargos y actividades: En un mundo tan competitivo, los estudiantes de maestría en Negocios deben promover en las estructuras más altas de las organizaciones para las cuales trabajan actividades de investigación y desarrollo con el fin de buscar mejores espacios de posicionamiento en ese campo. Promover líneas de investigación y desarrollo puede representar en el corto o mediano plazo un espacio más de crecimiento y satisfacción personal, entre otros.

- Método de selección de personal: En el mundo tan tecnológico, es importante que como nuevos profesionales estén abiertos a variados métodos de selección 
de personal como lo son la selección en línea, cuya técnica consiste básicamente en una presentación o grabación virtual por cualquier medio electrónico.

- Medio de búsqueda de empleo: Para asegurarse el éxito dentro del mercado laboral costarricense es importante que los actuales estudiantes diversifiquen su campo de acción mediante especializaciones técnicas. Dado el éxito en los medios de búsqueda de trabajo como las bolsas de empleo y contacto personales, nunca está sobrado, para quienes desean mejorar su expectativa laboral, participar activamente en ferias y ascensos internos dentro de las empresas. Igualmente, merece considerarse la oportunidad de incrementar cada vez más el espíritu empresarial y desarrollar un negocio propio que le asegure una mejor calidad de vida.

- Ingreso / formación: Con el fin de mejorar los niveles de ingresos de los estudiantes de maestría de Negocios de ULACIT, se debe buscar un equilibrio entre estudios y años de experiencia; es decir, evitar la acumulación de títulos universitarios sin sentido y buscar otras posibilidades en carreras técnicas de formación claves que exige el mercado laboral. Asimismo, promoverse internamente en la organización donde trabaja, aumentar su liderazgo y creatividad, diversificar su trabajo relacionado a la carrera universitaria, buscar opciones de trabajo en el sector privado, mejorar el trabajo en equipo para involucrarse con nuevas tareas que pueden ser una ventana al futuro, así como el posible desarrollo de un negocio propio.
Algunas líneas de investigación que pueden surgir de la presente investigación son:

Llevar a cabo un estudio de seguimiento de personas graduadas del programa de maestría de Negocios de la Universidad Latinoamericana de Ciencia y Tecnología (ULACIT) que responda a los intereses de los involucrados en este mercado laboral.

Realizar un análisis comparativo entre los datos obtenidos de $58 \%$ de hombres y $42 \%$ de mujeres con el fin de registrar las mejoras obtenidas entre las brechas existentes.

\section{Referencias}

Instituto Nacional de Estadística y Censos (2012). Encuesta continua de empleo de Costa Rica: Población según indicadores generales de la condición de actividad. Recuperado el 01-09-2012 de: http://www.inec.go.cr/Web/ Home/GeneradorPagina.aspx

Lobo, N., Morua, M. (2011) Un desafío innovador con impacto social Estudio de seguimiento a graduados de seis programas de posgrado de la UNED, con indicadores de calidad académica y mejoramiento continuo. Revista Posgrado y Sociedad, 11(1), pp.50-75

Lobo, N. (2011). Estudio de seguimiento de las personas graduadas del período 2001-2008 de trece programas de posgrados de las universidades estatales costarricenses. Costa Rica: Conare. Recuperado el 01-09-2012 de: www.posgrado.una.ac.cr/descargas/Est_Seg_Grad_ Posg_2009.pdf

Manpower. (2012). Encuesta sobre escasez de talento 2012. Recuperado el 20-09-2012, de: http://www.manpowergroup.com.mx/index.php/estudios/estudios-einvestigaciones

Morua, M. (2012). Contribuciones científicas de Schomburg y Teichler acerca del "mundo del trabajo"y su aplicación en estudios de mercado laboral para estudiantes de maestría en negocios que trabajan y estudian a la vez. Recuperado el 06-11-2012 de: Entrevista telefónica. 
RNA Revista Nacional de Administración

Schomburg, H. (2004). Manual para Estudios de Seguimiento de Graduados Universitarios. Recuperado el 01-11-2012 de: http://daad.csuca.org/attachments/146_manual_ es.pdf

Teichler, U. (2005). Graduados y empleo: investigación, metodología y resultados, los casos de Europa, Japón, Argentina y Uruguay. Buenos Aires, Argentina. Argentina: Miño y Dávila.

Recibido: 6 de marzo de 2013

Aceptado: 10 de abril de 2013 